U. S. DEPARTMENT OF THE INTERIOR

GEOLOGICAL SURVEY

\title{
ASSESSMENT OF CHEMICAL VARIABILITY IN THREE \\ INDEPENDENTLY PREPARED BATCHES OF NATIONAL \\ INSTITUTE FOR STANDARDS AND TECHNOLOGY SRM 2704, \\ BUFFALO RIVER SEDIMENT
}

S.A. $\stackrel{\text { By }}{\text { Wilson }}{ }^{1}$

Open File Report 93-692

This report is preliminary and has not been reviewed for conformity with U.S. Geological Survey editorial standards nor with the North American stratigraphic code. Any use of trade names is for descriptive purposes only and does not imply endorsement by the USGS.

1 U.S. Geological Survey, PO Box 25046, MS 973, Denver Co. 80225 


\section{CONTENTS}

Page

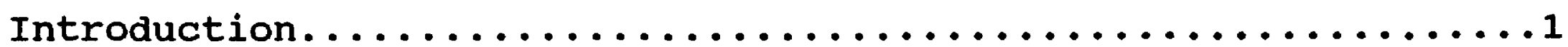

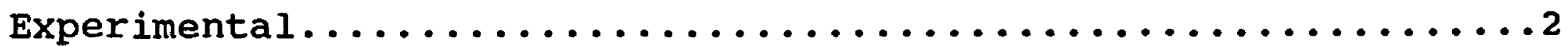

Results and Discussion ............................

Conclusions....................................

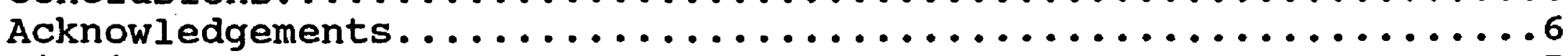

Bibliography ..................................

\section{TABLES}

Table 1. Comparison of USGS values for SRM 2704-original with NIST certified or recommended concentrations .......8

Table 2. Summary results for SRM 2704-original and

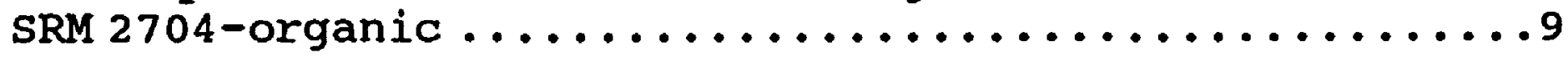

Table 3. Summary results for SRM 2704-organic and SRM 2704-

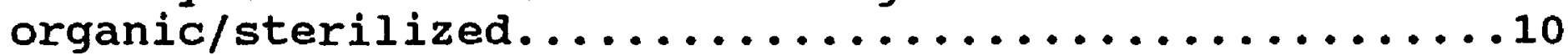

\section{APPENDICES}

Appendix A. Analytical results for SRM 2704-original, analysis by ICP-AES, WDXRF, HG-AAS and CV-AAS ...........11

Appendix B. Analytical results for SRM 2704-organic, analysis bY ICP-AES, WDXRF, HG-AAS, CV-AAS .............. 13

Appendix C. Analytical results for SRM 2704-organic/sterilized analysis by ICP-AES, WDXRF, HG-AAS, and CV-AAS ....20

Appendix D. Analytical data for SRM 2704-organic from second test group, analysis by ICP-AES, WDXRF, HG-AAS,

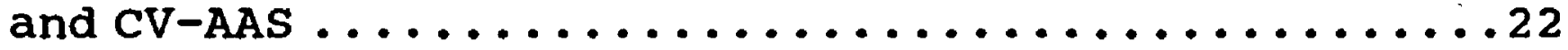




\section{INTRODUCTION}

Under a cooperative agreement between the National Institute for Standards and Technology (NIST) and the United States Geological survey (USGS), a study was conducted to determine the total element concentrations in three batches of standard reference material SRM 2704, also known as Buffalo River Sediment (National Institute for Standards and Technology, 1990). All three batches of SRM 2704 discussed in this report were derived from the same supply of the standard. The different SRM designations used in this report are designed to identify the different treatment procedures.

The first batch, identified as SRM 2704-original represents the currently distributed material that is certified for 25 major and trace elements. This supply of the standard was radiation sterilized immediately prior to bottling to stabilize the inorganic constituents against potential bacterial/microbial degradation. The second batch identified as SRM 2704-organic represents a supply of the original material that was destined to be certified for organic contaminates. SRM 2704-organic was not sterilized due to concerns over possible organic compound degradation by radiation sterilization. Comparisons of total element concentrations for these two batches of SRM 2704 was requested in order to determine if bottles of SRM 2704-organic could be added to the reserves of SRM 2704-original and help extend the lifetime of this valuable international reference material. If between batch comparisons of total element concentrations showed significant differences, then valuable information could be obtained regarding the long term stability of elements in a nonsterilized material.

The third batch of SRM 2704 used in this study was identified as 2704-organic/sterilized. This batch represents a separate split of 2704 -organic that had undergone radiation sterilization just prior to shipment of the samples. In this phase of the study a comparison was made between 2704-organic and 2704-organic/sterilized to determine if radiation sterilization has an effect on total element concentrations. of particular interest was the effect radiation sterilization would have on potentially volatile elements such as, arsenic, cadmium, mercury, and selenium.

In addition to the between batch comparison studies cited above, analytical results from the analysis of SRM 2704-original were also examined to evaluate the accuracy of USGS procedures. Comparison of USGS results with NIST certified values would be used to determine if a statistically significant bias existed for specific elements using USGS analytical procedures. 


\section{EXPERIMENTAL}

In phase one of the study, samples from SRM 2704-original and 2704-organic were randomly analyzed (in single job) to evaluate the between batch differences. Analytical results for SRM 2704-organic are based on the analysis of 25 individual bottles and 3 bottle duplicates $(n=28)$. Results for SRM 2704original are based on the analysis of 5 individual bottles and 2 bottle duplicates $(n=7)$. Approximately 1 to $7 \mathrm{~g}$ of material from each bottle of SRM was transferred to 2-ounce glass bottles and dried for two hours at $110^{\circ} \mathrm{C}$. Following the two hour drying time the bottles were immediately transferred to a desiccator to cool. After cooling the samples were removed from the desiccator, capped, and forwarded to the lab for analysis.

In phase two of the study, comparisons were made between 2704-organic and 2704-organic/sterilized. For this comparison, 5 bottles of SRM 2704-organic/sterilized were sampled along with 2 bottle duplicates $(n=7)$. Results for 2704/organic were based on samples from 3 individual bottles $(n=3)$. Samples were dried prior to analysis as described above. All total element concentrations are reported on a dry-weight basis.

All samples were analyzed using a combination of inductively coupled plasma-atomic emission spectrometry (ICP-AES), wavelength dispersive $X$-ray fluorescence spectroscopy (WDXRF), hydride generation-atomic absorption spectrophotometry (HG-AAS), and cold vapor-atomic absorption spectrophotometry (CV-AAS). The procedures used have been described in detail elsewhere (Arbogast, 1990; Baedecker, 1987; Crock, and others 1983) and will only be summarized here.

The ICP-AES procedure utilizes a $0.200-g$ sample which is transferred to a $30-\mathrm{mL}$ teflon beaker and $50 \mu \mathrm{g}$ of lutetium $\left(\mathrm{Lu}_{2} \mathrm{O}_{3}\right.$ in 5 percent $\mathrm{HCl}$ solution) added as an internal standard. The sample is digested to dryness overnight using concentrated $\mathrm{HCl}$ ( 3 $\mathrm{mL}), \mathrm{HNO}_{3}(2 \mathrm{~mL}), \mathrm{HClO}_{4}(1 \mathrm{~mL})$, and $\mathrm{HF}(2 \mathrm{~mL})$. The residue is redigested using $\mathrm{HClO}_{4}(1 \mathrm{~mL})$, and water at $150^{\circ} \mathrm{C}$, and taken to dryness again. One milliliter of aqua regia is finally added to the container, and the sample is diluted to $10.00 \mathrm{~g}$ with 1 percent $\mathrm{HNO}_{3}$. The solution is then analyzed for 40 elements simultaneously using a Jarrell Ash model 1160 spectrometer.

In WDXRF analyses, a $0.8000 \pm 0.004 \mathrm{~g}$ sample is ignited in a tared platinum crucible at $925^{\circ} \mathrm{C}$ for 45 minutes. The weight loss is reported as percent loss on ignition (\% LOI). An $8.00 \mathrm{~g}$ charge of lithium tetraborate is then added to the crucible along with a $0.250 \mathrm{~mL}$ aliquot of a 50 percent solution of lithium bromide, which serves as a nonwetting agent. Up to 7 crucibles are then placed on an "automatic fluxer," and the entire unit inserted into a muffle furnace, where the sample is fused for 40 
minutes at $1120^{\circ} \mathrm{C}$. After the fusion is complete the fluxing unit is removed from the muffle furnace, and the molten samples poured into specially designed two-piece platinum molds. When cooled, the individual glass disc is analyzed for its major element constituents using a Phillips model $1606 \mathrm{X}$-ray spectrometer. Major element concentrations are determined using calibration curves compiled from approximately 70 international geologic reference materials.

In the analysis of arsenic and selenium, $0.25 \mathrm{~g}$ of material are transferred to a 30-mL teflon bomb and moistened with $1 \mathrm{~mL}$ of 1 percent $\mathrm{HNO}_{3}$. The sample is digested overnight at $105^{\circ} \mathrm{C}$ using concentrated $\mathrm{HNO}_{3}(9 \mathrm{~mL}), \mathrm{HClO}_{4}(6 \mathrm{~mL})$, and $\mathrm{HF}(10 \mathrm{~mL})$. Following overnight digestion, $25 \mathrm{~mL}$ of 50 percent $(\mathrm{v} / \mathrm{v}) \mathrm{HCl}$ is added to the container and the solution allowed to stand for 30 minutes. The solution is then transferred to a $60-\mathrm{mL}$ polyethylene bottle and the mass adjusted to $54 \mathrm{~g}$ with deionized water. Samples are analyzed for arsenic and selenium using a Perkin Elmer 4100 and 303 atomic absorption spectrophotometers respectively. Both instruments are equipped with specially designed continuous-flow systems and the instruments are calibrated with commercially available aqueous standards. The procedure has a percent relative standard deviation (\%RSD) of approximately 10 percent (Crock, J.G., and Lichte, F.E., 1982 ; Sanzolone and Chao, 1987).

Total mercury concentration in the three batches of SRM 2704 was determined using a cold vapor-atomic absorption spectrophotometric procedure (CV-AAS). In this procedure, (Kennedy and Crock, 1987) $0.100 \mathrm{~g}$ of sample is transferred to a $16 \times 100-\mathrm{mm}$ glass test tube and $0.5 \mathrm{~mL}$ of sodium dichromate (25 percent $\mathrm{w} / \mathrm{v}$ ) and $2 \mathrm{~mL}$ of concentrated nitric acid added. The tube is then transferred to an aluminum heating block and the sample digested for 3 hours at $110^{\circ} \mathrm{C}$. Following digestion, the sample is cooled and the volume adjusted to $12 \mathrm{~mL}$ with deionized water. Samples are then analyzed on a Perkin Elmer model 303 atomic absorption spectrophotometer equipped with a USGS designed continuous-flow sample delivery system. Routine analysis of reference materials reveals that the method has a percent relative standard deviation of 10 percent.

\section{RESULTS AND DISCUSSION}

Prior to the comparison of between batch data, results from the USGS analysis of SRM 2704-original were compared with NIST certified values to evaluate the accuracy of USGS procedures. Results for 2704-original reported on a dry weight basis are presented in Appendix A. A summary of SRM 2704-original results along with NIST certified and noncertified values are reported in table 1. Using statistical guidelines published by NIST (Becker, 
D., and others, 1992), USGS results were compared to certified values to test the hypothesis that the mean total element concentrations are equal at the 95 percent confidence level. Comparison of WDXRF results for 9 major elements with the corresponding NIST values reveals that we can accept the hypothesis that the mean concentrations are the same at the 95 percent confidence level. A similar conclusion is reached for major elements quantified by the ICP-AES procedure, except for titanium which shows a significant bias (low) compared to the NIST value.

Comparison of ICP-AES trace element results with the NIST certified values reveals that only in the cases of arsenic, chromium, and cobalt is the hypothesis that the means are equal rejected at the 95 percent confidence level. Arsenic is also found to be biased (low) by the HY-AAS technique, though this observed concentration compares favorably with the ICP-AES value. A comparison of USGS mercury results using the CV-AAS procedure with the NIST certified value reveals the mean values are equal at the 95 percent confidence level.

One explanation for the lower concentrations of arsenic by the ICP-AES and HG-AAS procedures is that the three batches of SRM 2704 used in this study were oven dried according to the NIST protocol prior to analysis. It is possible that at the drying temperature of $110^{\circ} \mathrm{C}$, a portion of the arsenic was volatilized, leading to the lower concentrations observed in this study. Analysis of undried SRM 2704 was not possible under the guidelines of this study.

Comparison of NIST noncertified values with ICP-AES values shows general agreement except for cerium, where the USGS value appears to be significantly lower. Based on the good agreement between USGS and NIST total element concentrations, it is reasonable to assume that USGS analytical procedures provide an accurate estimation of total element concentrations in SRM 2704 .

In phase one of the study, data from SRM 2704-original were compared against results for SRM 2704-organic to determine if significant differences in total element concentrations existed between the two batches of standards. Because it was unclear at the start of this study if SRM 2704-organic had ever been examined for homogeneity with respect to its inorganic constituents, a test was performed to evaluate the between- and within-bottle variability. The homogeneity evaluation for SRM 2704-organic was based on the analysis of 25 individual bottles and 3 bottle replicates $(n=28)$. Individual results corrected to dry weight are reported in Appendix B. Results for ICP-AES and WDXRF analyses were evaluated using an analysis of variance (ANOVA). The ANOVA revealed no significant difference for between- or within-bottle total element concentrations at the 95 percent confidence level. Two exceptions were ICP-AES results for 
aluminum and nickel. In the case of aluminum, conclusions based on ICP-AES results are not substantiated by the WDXRF data, which show no statistical difference in the data sets. In the case of nickel, even though the means are statistically different, the fact that both means fall within the NIST 95 percent confidence interval and that the relative difference in mean concentrations is less than 3 percent suggests that from a practical standpoint the values should be considered the same.

After establishing that no significant between-bottle differences existed for SRM 2704-organic, a comparison of total element concentrations was made between 2704-original and 2704organic. Differences in mean total element concentrations were evaluated using a two sample t-test (Walpole, R.E. and Myers, R.H. , 1989) . A comparison of average total element concentrations is summarized in table 2 along with an indication if there was ( $Y$ ) or was not (N) a statistical difference in mean total element concentrations at the 95 percent confidence level. of the 26 trace elements evaluated, a total of 11 (42 percent) show a statistically significant difference in mean total element concentrations for the two batches of SRM 2704. Most notable are the difference observed for chromium, lead, and zinc by ICP-AES, arsenic by CV-AAS, and mercury by CV-AAS. Conclusions regarding the between batch differences for arsenic and mercury should be viewed with caution, because of the drying step used in the sample preparation and the possibility of element volatilization. While element volatilization is a distinct possibility, it should also be pointed out that the two batches were randomly combined and dried simultaneously under identical conditions. If volatilization did occur, it is reasonable to assume that all samples would be affected equally.

In the case of the major elements, where both ICP-AES and WDXRF results are available, conclusions regarding between batch differences are less definitive. For magnesium, phosphorous, and titanium, both ICP-AES and WDXRF results indicate that there is no statistical difference in the mean concentrations at the 95 percent confidence level. In case of aluminum, calcium, potassium, and sodium, conclusions regarding between batch differences in mean total element concentrations are unclear because of the conflicting statistical results. Only in the case of iron do both techniques indicate that the two batches are statistically different.

In phase two of the study samples of SRM-organic and SRMorganic/sterilized were compared to determine if radiation sterilization affects total element concentrations. In a separate experiment, samples from five bottles of SRM 2704-organic/ sterilized supplied by NIST were randomly analyzed along with samples from three bottles of SRM 2704-organic. A statistical summary of the data is presented in table 3 and individual values corrected to dry weight are presented in Appendices $C$ and D. 
Average concentrations and standard deviations for 2704organiclsterilized represent a combination of between and within bottle results $(n=7)$. Average concentrations and standard deviations for 2704-organic are based solely on the analysis of between bottle determinations $(n=3)$. Comparison of mean total element concentrations for 2704-organic and 2704-organic/ sterilized using a pooled t-test reveals that for the majority (89 percent) of elements mean total element concentrations are considered equal at the 95 percent confidence level. Exceptions include sodium, magnesium, and calcium by ICP-AES, and potassium by WDXRF.

\section{CONCLUSIONS}

Comparison of analytical results for SRM 2704-original and SRM 2704-organic indicates that for the majority of elements there is no significant difference in mean total element concentration at the 95 percent confidence level. Where statistical differences do exist, the lower concentrations are normally associated with SRM 2704-organic, suggesting that unsterilized material can change over time. It is also apparent from the excellent agreement between USGS and NIST certified values for SRM 2704-original that once the material has been radiation sterilized it is stable over time. The effect of radiation sterilization on total element concentration on SRM 2704 is found to be negligible as evidenced by the similarity in analytical results for 2704-organic and 2704-organic/sterilized. This is especially significant for volatile elements such as mercury, arsenic, selenium, and cadmium. Before a final conclusion is reached regarding the between batch differences observed in this study, NIST scientists need to evaluate the magnitude of these difference with respect to the recommended confidence interval for each element.

\section{ACKNOWLEDGEMENTS}

This work was supported by NIST under contract order number 43NANB312718. Completion of this study could not have been possible with out the contributions of P. Briggs, D. Siems, J. S. Mee, P. Hageman, and E. Welsch. 


\section{BIBLIOGRAPHY}

Arbogast, B.F., (ed.), 1990, Quality Assurance Manual for the Branch of Geochemistry, U.S. Geological Survey, 184 p.

Baedecker, P.A., (ed.), 1987, Methods of Geochemical Analysis, U.S. Geological Survey Bulletin 1770, $180 \mathrm{p}$.

Becker, D., Christensen, R., Currie, L., Diamondstone, B., Eberhardt, K., Gills, T., Hertz, H., Klouda, G., Moody, J., Parris, R., Schaffer, R., Steel, E., Taylor, J., Watters, R., Zeisler, R., 1992, Use of NIST Standard Reference Materials for Decisions on Performance of Analytical Chemical Methods and Laboratories, NIST Special Publication $829,27 p$.

Crock, J.G., and Lichte, F.E., (1982), An improved method for the determination of trace levels of arsenic an antimony in geologic materials by automated hydride generation-atomic absorption spectroscopy, Analytica Chimica Acta, 144, 223-33

Crock, J.G., Lichte, F.E., and Briggs, P.H., 1983 Determination of elements in National Bureau of Standards' geologic reference materials SRM 278 obsidian and SRM 688 Basalt by Inductively Coupled Argon Plasma-Atomic Emission Spectroscopy, Geostandards Newsletter, 7, 335-340

Kennedy, K.R., and Crock, J.G., 1987, Determination of mercury in geological material by continuous flow, cold-vapor, atomic absorption spectrophotometry, Analytical Letters, 20, 899908

National Institute for Standards and Technology, 1990 Certificate, Standard Reference Material 2704, Buffalo River sediment.

Sanzolone R.F. and Chao, T.T., 1987, Determination of selenium in thirty-two geochemical reference materials by continuousflow hydride generation atomic absorption spectrophotometry, Geostandards Newsletter, 11, 81-85.

Walpole R.E., and Myers, R.H., 1989, Probability and Statistics for Engineers and Scientists, 4th ed. MacMillan Publishing Company, New York, 765 p. 
Table 1. Comparison of USGS values for SRM 2704-original with NIST certified or recommended concentrations

\begin{tabular}{|c|c|c|c|c|c|}
\hline & USGS & $\begin{array}{l}\text { USGS } \\
\text { Average }\end{array}$ & & $\begin{array}{c}\text { NIST } \\
\text { Certified }^{3}\end{array}$ & \\
\hline Element & Method & conc, 8 & $\underline{S T D^{2}}$ & conc, of & $\underline{S_{T D}^{4}}$ \\
\hline $\mathrm{Al}$ & ICP & 6.14 & 0.03 & 6.11 & 0.16 \\
\hline Al & WDXRF & 6.19 & 0.12 & & \\
\hline $\mathrm{Ca}$ & ICP & 2.65 & 0.02 & 2.60 & 0.03 \\
\hline $\mathrm{Ca}$ & WDXRF & 2.62 & 0.01 & & \\
\hline $\mathrm{Fe}$ & ICP & 4.08 & 0.03 & 4.11 & 0.10 \\
\hline $\mathrm{Fe}$ & WDXRF & 4.14 & 0.01 & & \\
\hline $\mathrm{K}$, & ICP & 1.95 & 0.01 & 2.00 & 0.04 \\
\hline $\mathbf{K}$, & WDXRF & 2.01 & 0.01 & & \\
\hline $\mathrm{Mg}$ & ICP & 1.23 & 0.02 & 1.20 & 0.02 \\
\hline $\mathrm{Mg}$ & WDXRF & 1.20 & 0.01 & & \\
\hline $\mathrm{Na}$ & ICP & 0.60 & 0.01 & 0.547 & 0.014 \\
\hline $\mathrm{Na}$ & WDXRF & 0.56 & 0.01 & & \\
\hline P, & ICP & 0.10 & $<0.005$ & 0.0998 & $0.002 \varepsilon$ \\
\hline P, & WDXRF & 0.10 & $<0.005$ & & \\
\hline $\mathbf{s i}$ & WDXRF & 29.15 & 0.12 & 29.08 & 0.13 \\
\hline $\mathbf{T i}$ & ICP & 0.40 & 0.02 & 0.457 & 0.018 \\
\hline $\mathbf{T i}$ & WDXRF & 0.46 & $<0.005$ & & \\
\hline
\end{tabular}

\begin{tabular}{|c|c|c|c|c|c|}
\hline$\frac{\text { Element }}{A s}$ & $\frac{\text { Method }}{\text { ICP }}$ & $\frac{\mu \mathrm{g} / \mathrm{g}}{20}$ & $\frac{\text { STD }}{1.4}$ & $\frac{\mu \mathrm{g} / \mathrm{g}}{23.4}$ & $\frac{\mathrm{STD}}{0.8}$ \\
\hline As & HY-AAS & 20 & 0.5 & & \\
\hline $\mathrm{Ba}$ & ICP & 412 & 4 & 414 & 12 \\
\hline $\mathrm{Cd}$ & ICP & 3 & 0.5 & 3.45 & 0.22 \\
\hline $\mathrm{Cr}$ & ICP & 143 & 2 & 135 & 5 \\
\hline Co & ICP & 16 & 0.5 & 14 & 0.6 \\
\hline $\mathrm{Cu}$ & ICP & 97 & 1 & 98.6 & 5 \\
\hline $\mathrm{Hg}$ & CV-AAS & 1.4 & 0.05 & 1.44 & 0.07 \\
\hline Mn & ICP & 575 & 8 & 555 & 19 \\
\hline $\mathbf{N i}$ & ICP & 44 & 1 & 44.1 & 3.0 \\
\hline $\mathbf{P b}$ & ICP & 157 & 6 & 161 & 17 \\
\hline $\mathrm{v}$, & ICP & 90 & 1 & 95 & 4 \\
\hline $\mathrm{zn}$ & ICP & 429 & 9 & 438 & 12 \\
\hline
\end{tabular}

\begin{tabular}{|c|c|c|c|c|}
\hline Element & $\begin{array}{l}\text { USGS } \\
\text { Method }\end{array}$ & $\mu \mathrm{g} / \mathrm{g}$ & STD & $\begin{array}{l}\text { ommended } \\
\text { conc. } \\
\mu \mathrm{g} / \mathrm{q}\end{array}$ \\
\hline $\mathrm{Ce}$ & ICP & 57 & 2 & 72 \\
\hline La & ICP & 32 & 0.5 & 29 \\
\hline$\vec{L} \mathbf{i}$ & ICP & 49 & 0.5 & 50 \\
\hline Sc & ICP & 12 & $<0.5$ & 12 \\
\hline $\mathrm{Se}$ & HY-AAS & 1.0 & $<0.05$ & 1.1 \\
\hline Sr & ICP & 136 & 1 & 130 \\
\hline Th & ICP & 9 & 1 & 9.2 \\
\hline $\mathrm{Yb}$ & ICP & 2 & $<0.5$ & 2.8 \\
\hline
\end{tabular}

1 Average based on seven determinations

2 One standard deviation

3 See certificate for definition

4 NIST's calculated 95 percent confidence interval 
Table 2. Summary results for 2704-original and SRM 2704-organic

\begin{tabular}{|c|c|c|c|c|c|c|c|}
\hline & & & $\begin{array}{l}\text { SRM } \\
\text { ori }\end{array}$ & $\begin{array}{l}2704 \\
\text { inal }\end{array}$ & & $\begin{array}{c}2704 \\
\text { anic }\end{array}$ & $\begin{array}{r}\text { Signif } \\
\text { Diff. }\end{array}$ \\
\hline Elen & nent & Method & Avg. ${ }^{\prime}$ & STD. ${ }^{2}$ & Avg. ${ }^{3}$ & STD. ${ }^{2}$ & $\underline{Y / N^{4}}$ \\
\hline$\overline{\mathrm{Al}}$, & 8 & ICP & 6.14 & 0.03 & 6.09 & 0.03 & $\mathbf{N}$ \\
\hline Al, & 8 & WDXRF & 6.19 & 0.03 & 6.16 & 0.03 & $\mathbf{Y}$ \\
\hline $\mathrm{Ca}$, & 8 & ICP & 2.65 & 0.02 & 2.66 & 0.02 & $\mathbf{N}$ \\
\hline $\mathrm{Ca}$, & 8 & WDXRF & 2.62 & 0.01 & 2.64 & 0.01 & $\mathbf{Y}$ \\
\hline $\mathrm{Fe}$, & 8 & ICP & 4.08 & 0.03 & 3.99 & 0.02 & $Y$ \\
\hline $\mathrm{Fe}$, & 8 & WDXRF & 4.14 & 0.01 & 4.06 & 0.02 & $\mathbf{Y}$ \\
\hline $\mathrm{K}$, & 8 & ICP & 1.95 & 0.01 & 1.94 & 0.01 & $Y$ \\
\hline & 8 & WDXRF & 2.01 & 0.01 & 2.00 & 0.01 & $\mathbf{N}$ \\
\hline Mg, & 8 & ICP & 1.23 & 0.02 & 1.22 & 0.01 & $\mathbf{N}$ \\
\hline Mg, & 8 & WDXRF & 1.20 & 0.01 & 1.20 & 0.01 & $\mathbf{N}$ \\
\hline $\mathrm{Na}$, & 8 & ICP & 0.60 & 0.01 & 0.61 & 0.01 & $\mathbf{N}$ \\
\hline $\mathrm{Na}$, & 8 & WDXRF & 0.56 & 0.01 & 0.58 & 0.01 & $\mathbf{Y}$ \\
\hline P, & 8 & ICP & 0.10 & $<0.005$ & 0.10 & $<0.005$ & $\mathbf{N}$ \\
\hline P, & 8 & WDXRF & 0.10 & $<0.005$ & 0.10 & $<0.005$ & $\mathbf{N}$ \\
\hline si, & 8 & WDXRF & 29.14 & 0.12 & 29.23 & 0.10 & $\mathbf{N}$ \\
\hline$T i$, & 8 & $I C P$ & 0.40 & 0.02 & 0.40 & 0.02 & $\mathbf{N}$ \\
\hline $\mathrm{Ti}$, & 8 & WDXRF & 0.46 & $<0.005$ & 0.46 & $<0.005$ & $\mathbf{N}$ \\
\hline LOI, & 8 & WDXRF & 9.23 & 0.08 & 9.16 & 0.08 & $\mathbf{Y}$ \\
\hline As, & $\mu \mathrm{g} / \mathrm{g}$ & ICP & 20 & 1.4 & 15 & 1.2 & $\mathbf{Y}$ \\
\hline As, & $\mu \mathrm{g} / \mathrm{g}$ & HG-AAS & 20. & 1.0 & 16 & 0.6 & $Y$ \\
\hline $\mathrm{Ba}$, & $\mu \mathrm{g} / \mathrm{g}$ & ICP & 412 & 4.2 & 412 & 5.2 & $\mathbf{N}$ \\
\hline $\mathrm{Be}$, & $\mu g / g$ & ICP & 2 & $<0.5$ & 2 & $<0.5$ & $\mathbf{N}$ \\
\hline$c d$, & $\mu g / g$ & ICP & 3.3 & 0.5 & 3 & $<0.5$ & $\mathbf{Y}$ \\
\hline Ce, & $\mu \mathrm{g} / \mathrm{g}$ & ICP & 57 & 1.7 & 56 & 1.9 & $\mathbf{N}$ \\
\hline Co, & $\mu \mathrm{g} / \mathrm{g}$ & ICP & 16 & 0.5 & 15 & 0.6 & $\mathbf{N}$ \\
\hline Cr, & $\mu \mathrm{g} / \mathrm{g}$ & ICP & 143 & 2.0 & 128 & 2.5 & $\mathbf{Y}$ \\
\hline $\mathrm{Cu}$, & $\mu \mathrm{g} / \mathrm{g}$ & ICP & 97 & 1.2 & 92 & 5.0 & $\mathbf{Y}$ \\
\hline Ga, & $\mu \mathrm{g} / \mathrm{g}$ & ICP & 15 & 0.5 & 15 & 0.5 & $\mathbf{N}$ \\
\hline $\mathrm{Hg}$, & $\mu \mathrm{g} / \mathrm{g}$ & CV-AAS & 1.4 & 0.05 & 1.0 & 0.1 & $\mathbf{Y}$ \\
\hline La, & $\mu \mathrm{g} / \bar{g}$ & ICP & 32 & 0.5 & 32 & 1.1 & $\mathbf{N}$ \\
\hline Li, & $\mu \mathrm{g} / \mathrm{g}$ & ICP & 49 & 0.5 & 48 & 0.4 & $\mathbf{Y}$ \\
\hline Mn, & $\mu g / g$ & ICP & 575 & 7.5 & 563 & 5.1 & $\mathbf{Y}$ \\
\hline Nb, & $\mu \mathrm{g} / \overline{\mathrm{g}}$ & ICP & 9 & 1.3 & 8 & 0.7 & $\mathbf{N}$ \\
\hline Nd, & $\mu \mathrm{g} / \mathrm{g}$ & ICP & 29 & 1.2 & 30 & 1.2 & $\mathbf{N}$ \\
\hline Ni, & $\mu \mathrm{g} / \mathrm{g}$ & ICP & 44 & 1.1 & 43 & 1.9 & $\mathbf{N}$ \\
\hline $\mathrm{Pb}$ & $\mu \mathrm{g} / \mathrm{g}$ & ICP & 157 & 5.8 & 146 & 7.5 & $\mathbf{Y}$ \\
\hline sc, & $\mu \mathrm{g} / \mathrm{g}$ & ICP & 12 & $<0.5$ & 12 & $<0.5$ & $\mathbf{N}$ \\
\hline Se, & $\mu g / g$ & HG-AAS & 1 & $<0.05$ & 1 & $<0.05$ & $\mathbf{N}$ \\
\hline sr, & $\mu \mathrm{g} / \mathrm{g}$ & ICP & 136 & 1.1 & 137 & 1.1 & $\mathbf{N}$ \\
\hline Th, & $\mu \mathrm{g} / \mathrm{g}$ & ICP & 9 & 1.2 & 10 & 0.8 & $\mathbf{N}$ \\
\hline v, & $\mu \mathrm{g} / \mathrm{g}$ & ICP & 90 & 0.8 & 89 & 0.8 & $\mathbf{Y}$ \\
\hline Y, & $\mu g / g$ & ICP & 31 & 0.8 & 31 & 0.5 & $\mathbf{N}$ \\
\hline Yb, & $\mu \mathrm{g} / \mathrm{g}$ & ICP & 2 & $<0.5$ & 2 & 0.31 & $\mathbf{N}$ \\
\hline$\underline{\mathrm{z}}$, & $\mu q / q$ & ICP & 429 & 8.6 & 395 & 8.7 & $Y$ \\
\hline
\end{tabular}

1 Average based on 7 determinations

2 One Standard Deviation

3 Average based on 28 determinations

4 Average values are (Y), or are not (N), significantly different at the 95 percent confidence interval 
Table 3. Summary results for SRM 2704-organic and 2704-organic/sterilized

\begin{tabular}{|c|c|c|c|c|c|c|c|}
\hline & & & $\begin{array}{l}\text { SRM } 2 \\
\text { orgar }\end{array}$ & & $\begin{array}{r}\text { SRM } 27 \\
\text { organi } \\
\text { sterili }\end{array}$ & & $\begin{array}{l}\text { Signif. } \\
\text { Different }\end{array}$ \\
\hline Elem & ent & Method & Avg. 1 & STD. ${ }^{2}$ & Avg. ${ }^{3}$ & STD. ${ }^{2}$ & $(Y / N)^{4}$ \\
\hline Al, & 8 & $I C P$ & 6.10 & 0.05 & 6.08 & 0.05 & $\mathbf{N}$ \\
\hline Al, & 8 & WDXRF & 6.10 & 0.03 & 6.12 & 0.03 & $\mathbf{N}$ \\
\hline $\mathrm{Ca}$, & 8 & ICP & 2.63 & 0.01 & 2.59 & 0.02 & $\mathbf{Y}$ \\
\hline $\mathrm{Ca}$, & 8 & WDXRF & 2.63 & 0.01 & 2.63 & 0.01 & $\mathbf{N}$ \\
\hline $\mathbf{F e}$, & 8 & ICP & 4.15 & $<0.005$ & 4.11 & 0.04 & $\mathbf{N}$ \\
\hline Fe, & 8 & WDXRF & 4.07 & 0.01 & 4.08 & 0.04 & $\mathrm{~N}$ \\
\hline $\mathrm{K}$ & 8 & ICP & 1.99 & 0.09 & 2.02 & 0.03 & $\mathbf{N}$ \\
\hline & 8 & WDXRF & 1.99 & $<0.005$ & 2.00 & 0.01 & $\mathbf{Y}$ \\
\hline $\mathrm{Mg}$, & $\%$ & ICP & 1.26 & 0.01 & 1.25 & 0.01 & $\mathbf{Y}$ \\
\hline $\mathrm{Mg}$, & $q$ & WDXRF & 1.18 & 0.02 & 1.19 & 0.01 & $\mathbf{N}$ \\
\hline $\mathrm{Na}$, & 8 & ICP & 0.62 & $<0.015$ & 0.61 & 0.01 & $\Psi$ \\
\hline $\mathrm{Na}$, & 8 & WDXRF & 0.57 & $<0.005$ & 0.56 & 0.01 & $\mathbf{N}$ \\
\hline P, & $\%$ & ICP & 0.11 & $<0.005$ & 0.11 & $<0.005$ & $\mathbf{N}$ \\
\hline P, & $\%$ & WDXRF & 0.10 & $<0.005$ & 0.10 & $<0.005$ & $\mathbf{N}$ \\
\hline si, & 8 & WDXRF & 29.07 & 0.09 & 29.08 & 0.03 & $\mathbf{N}$ \\
\hline Ti, & 8 & ICP & 0.34 & 0.02 & 0.32 & 0.01 & $\mathbf{N}$ \\
\hline$T i$, & $\%$ & WDXRF & 0.45 & $<0.005$ & 0.46 & $<0.005$ & $\mathbf{N}$ \\
\hline LOI, & 8 & WDXRF & 9.12 & $<0.005$ & 9.08 & 0.06 & $\mathbf{N}$ \\
\hline As, & $\mu \mathrm{g} / \mathrm{g}$ & ICP & 22 & 1.2 & 20 & 1.8 & $\mathbf{N}$ \\
\hline As, & $\mu \mathrm{g} / \mathrm{g}$ & HG-AAS & 17 & 1.1 & 17 & 0.5 & $\mathbf{N}$ \\
\hline $\mathrm{Ba}$, & $\mu \mathrm{g} / \mathrm{g}$ & ICP & 414 & 2.6 & 409 & 4.3 & $\mathbf{N}$ \\
\hline $\mathrm{Be}$, & $\mu \mathrm{g} / \mathrm{g}$ & ICP & 2 & 0.6 & 2 & $<0.5$ & $\mathbf{N}$ \\
\hline Cd, & $\mu \mathrm{g} / \mathrm{g}$ & ICP & 3 & 0.07 & 3 & $<0.5$ & $\mathbf{N}$ \\
\hline $\mathrm{Ce}$, & $\mu \mathrm{g} / \mathrm{g}$ & ICP & 60 & 2.2 & 58 & 1. & $\mathbf{N}$ \\
\hline Co, & $\mu \mathrm{g} / \mathrm{g}$ & ICP & 15 & 0.3 & 15 & 0.4 & $\mathbf{N}$ \\
\hline $\mathrm{Cr}$, & $\mu \mathrm{g} / \mathrm{g}$ & ICP & 126 & 2.0 & 123 & 1.4 & $\mathbf{N}$ \\
\hline $\mathrm{Cu}$, & $\mu \mathrm{g} / \mathrm{g}$ & ICP & 92 & 2.9 & 92 & 4 & $\mathbf{N}$ \\
\hline Ga, & $\mu \mathrm{g} / \mathrm{g}$ & ICP & 15 & 0.2 & 15 & 0.7 & $\mathbf{N}$ \\
\hline $\mathrm{Hg}$, & $\mu \mathrm{g} / \mathrm{g}$ & CV-AAS & 1.0 & 0.08 & 1.1 & 0.17 & $\mathbf{N}$ \\
\hline La, & $\mu g / g$ & ICP & 29 & 0.7 & 29 & 0.5 & $\mathbf{N}$ \\
\hline Li, & $\mu \mathrm{g} / \mathrm{g}$ & ICP & 49 & 0.2 & 49 & 0.2 & $\mathbf{N}$ \\
\hline Mn, & $\mu \mathrm{g} / \mathrm{g}$ & ICP & 585 & 3 & 577 & 4.7 & $\mathbf{N}$ \\
\hline $\mathrm{Nb}$, & $\mu g / g$ & ICP & 9 & 0.5 & 9 & 0.5 . & $\mathbf{N}$ \\
\hline Nd, & $\mu \mathrm{g} / \mathrm{g}$ & ICP & 29 & 0.9 & 29 & 0.8 & $\mathbf{N}$ \\
\hline $\mathrm{Ni}$, & $\mu \mathrm{g} / \mathrm{g}$ & ICP & 42 & 0.3 & 42 & 1.8 & $\mathbf{N}$ \\
\hline $\mathbf{P b}$, & $\mu \mathrm{g} / \mathrm{g}$ & ICP & 148 & 8.7 & 144 & 4.7 & $\mathbf{N}$ \\
\hline Sc, & $\mu g / g$ & ICP & 12 & $<0.5$ & 12 & $<0.5$ & $\mathbf{N}$ \\
\hline Se, & $\mu \mathrm{g} / \mathrm{g}$ & HG-AAS & 1.0 & 0.06 & 1.1 & 0.04 & $\mathbf{N}$ \\
\hline Sr, & $\mu g / g$ & ICP & 139 & 1.2 & $13 \overline{6}$ & 1.3 & $\mathbf{N}$ \\
\hline Th, & $\mu g / g$ & ICP & 10 & 0.7 & 10 & 0.9 & $\mathbf{N}$ \\
\hline v, & $\mu \mathrm{g} / \vec{g}$ & ICP & 95 & 0.4 & 94 & 0.8 & $\mathbf{N}$ \\
\hline$Y$, & $\mu g / g$ & ICP & 24 & 0.1 & 24 & 0.2 & $\mathbf{N}$ \\
\hline Yb, & $\mu \mathrm{g} / \mathrm{g}$ & ICP & 2 & $<0.5$ & 2 & 0.2 & $\mathbf{N}$ \\
\hline$\underline{2 n}$, & $\mu \mathrm{g} / \mathrm{g}$ & ICP & 408 & 2.3 & 404 & 8.1 & N \\
\hline
\end{tabular}

1 Average value based on 3 determinations

2 One Standard Deviation

3 Average value based on 5 determinations

4 Values are (Y), or are not (N) significantly different at the 95 percent confidence interval 
h|

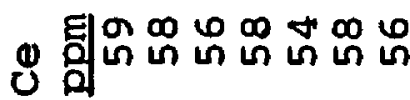

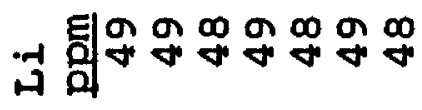

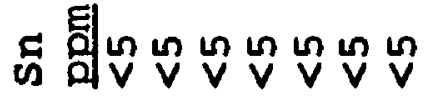

告

सी 0000000

0 网

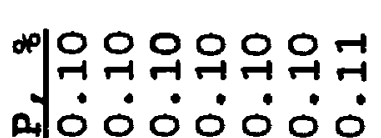

ช 맴mm

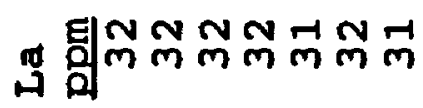

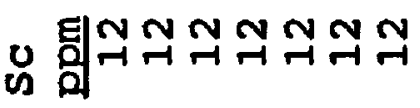

*|ำ

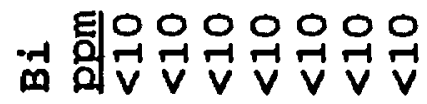

㞷吕吕

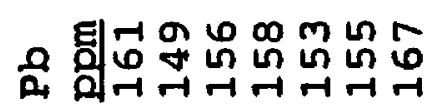

"m

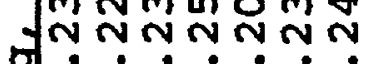

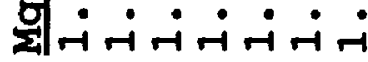

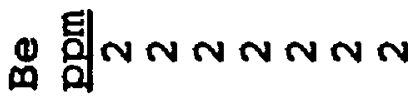

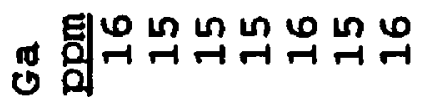

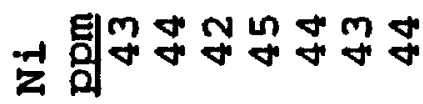

H

d \% $\sigma \sigma \sigma \sigma \sigma$

复

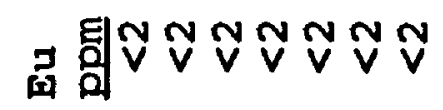

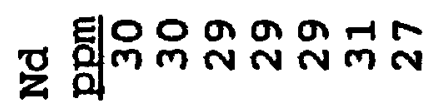

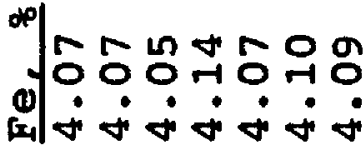
马

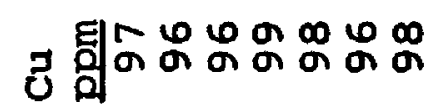

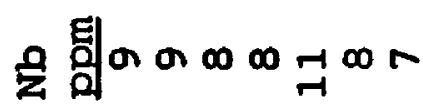

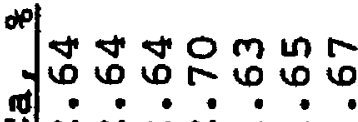

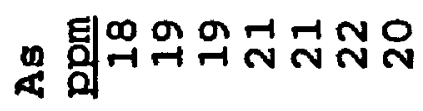

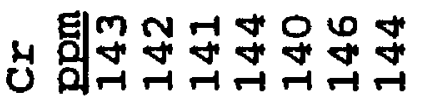

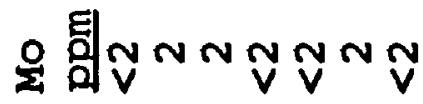

* هُ

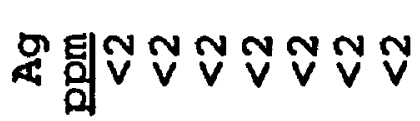

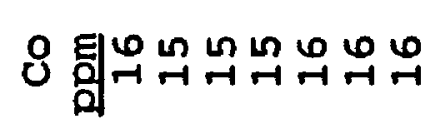

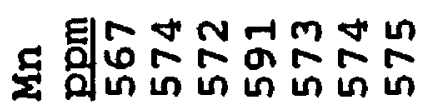

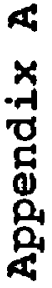

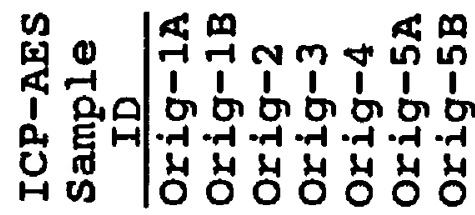

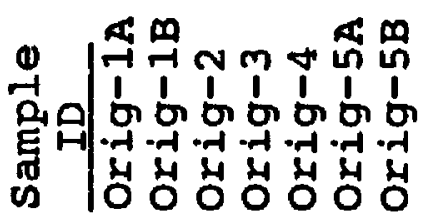

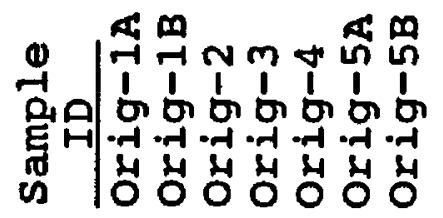

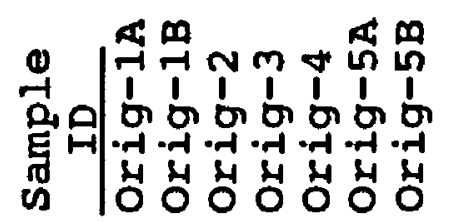




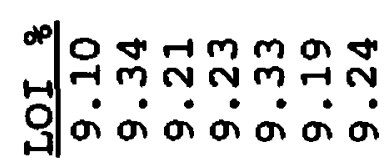

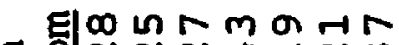

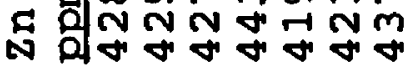

ค ด्ดी

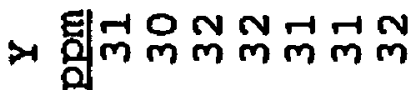

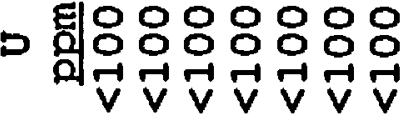

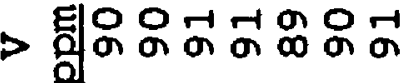

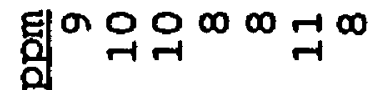

팅ㅇㅇㅇㅇㅇㅇㅇㅇㅇ은

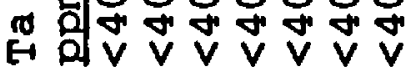

티에 6 un 00 แ मू مीmmmmmm

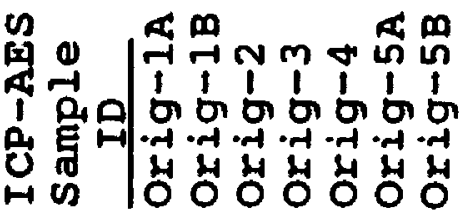

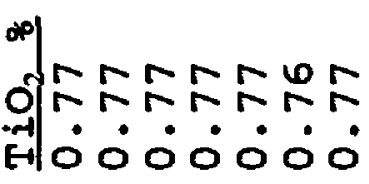

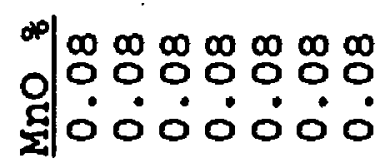

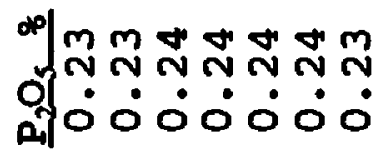

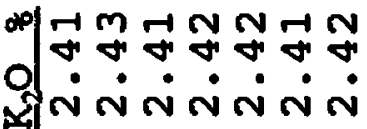

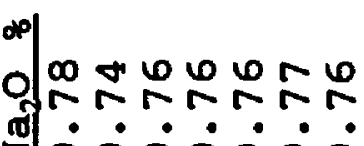

N

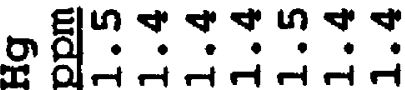

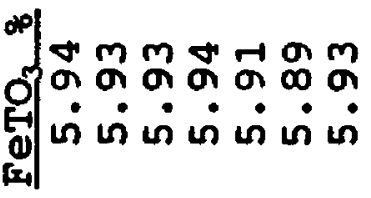

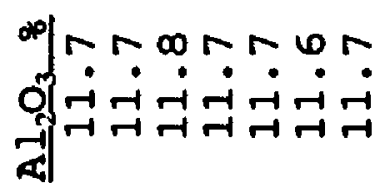

op mm MON om? .-1

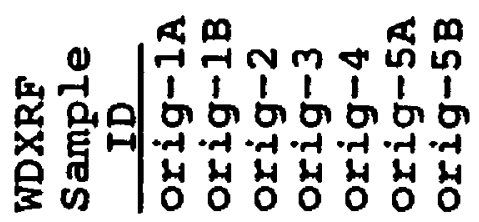

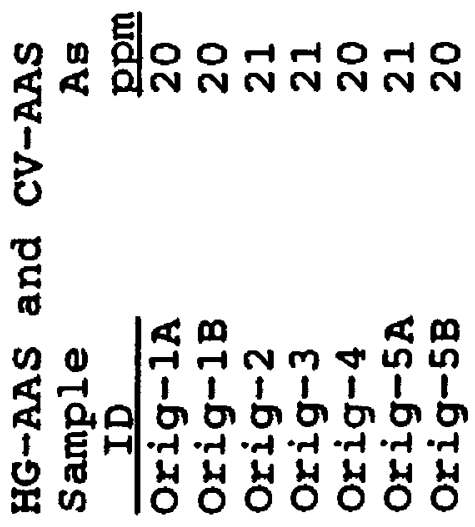




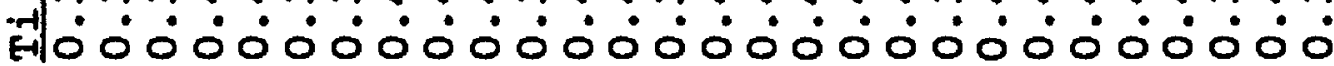

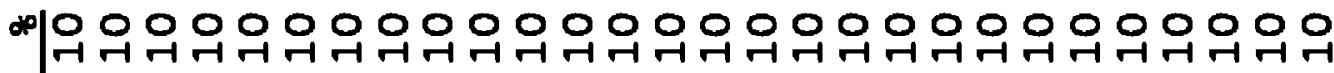

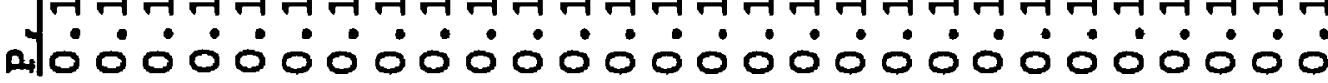

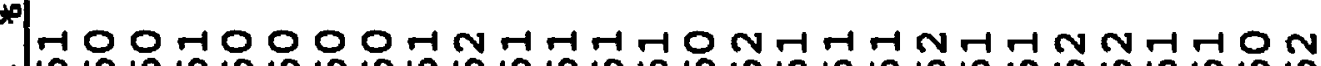
ก.

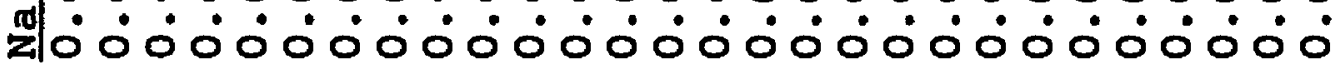

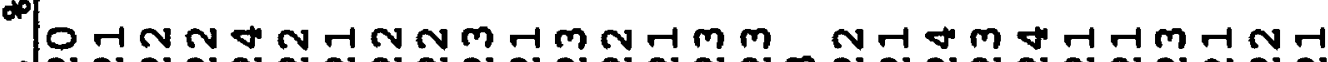
gnv

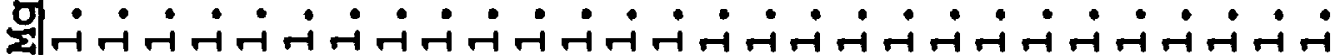

中mm $m$ m

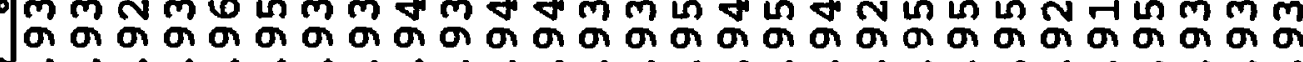

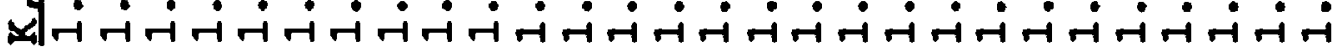

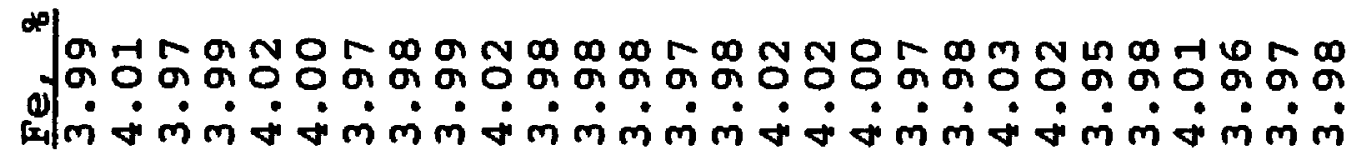

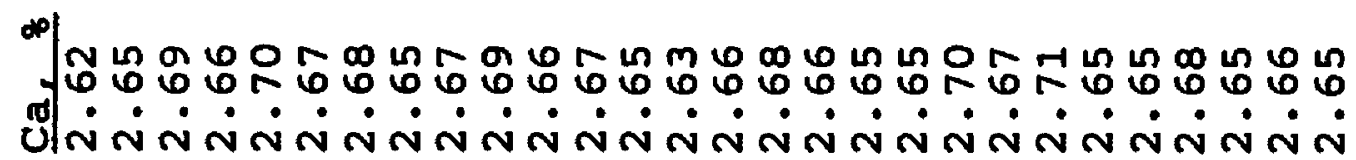

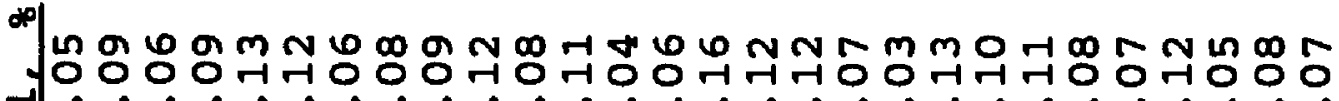

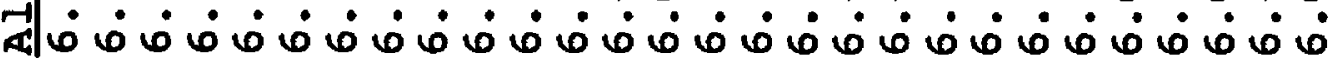


ఫึம

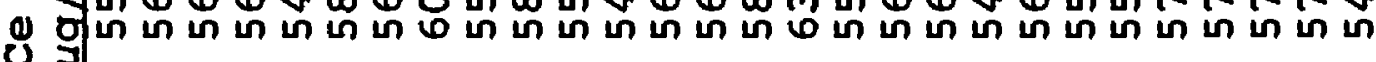
ఫु ఫึलmmmmmmmmmmmmmmmmmmmmmmmmmmm g]0000000000000000000000000000

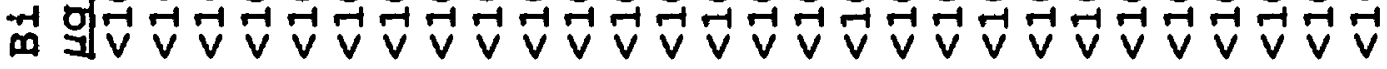

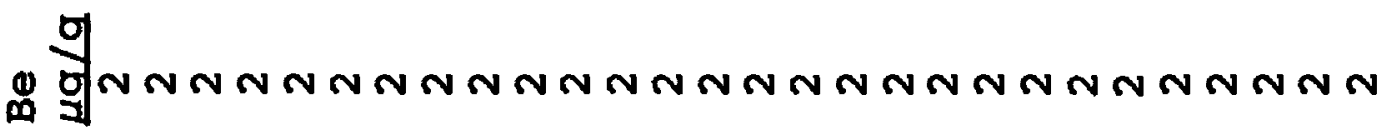

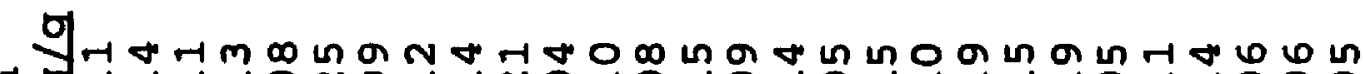
๓ 马 q

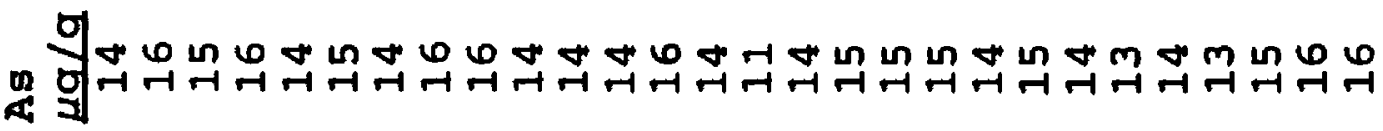

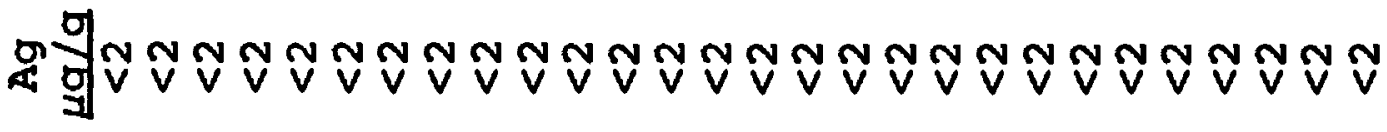

$\infty$

苟 no

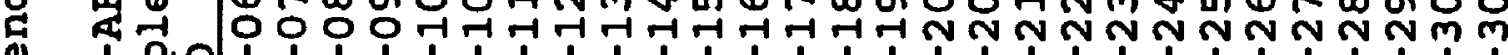
\&

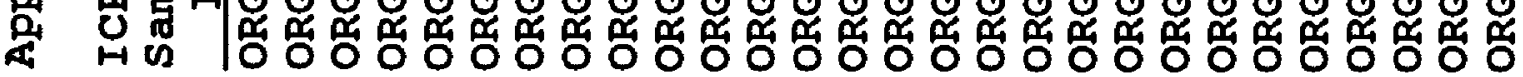




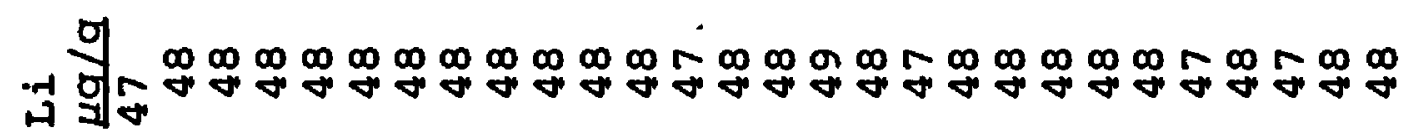

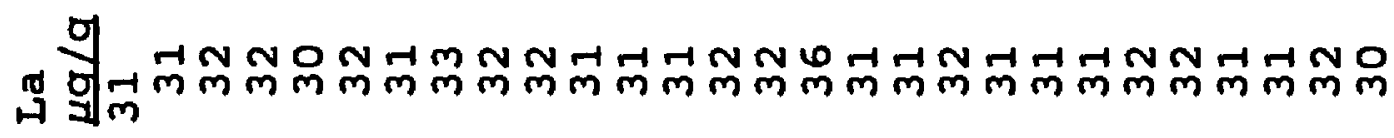

吽哥 ชู ำำ

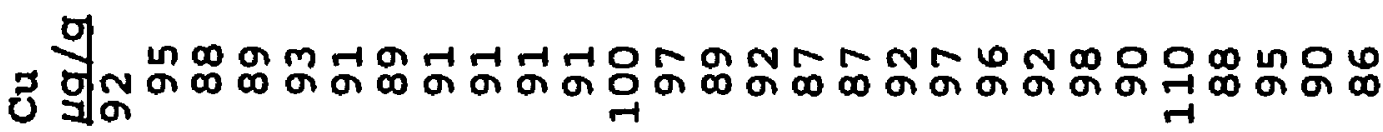

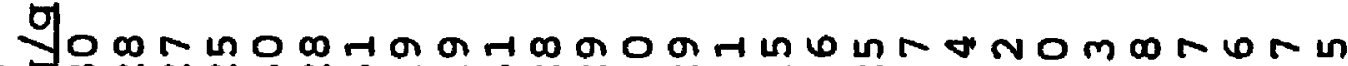

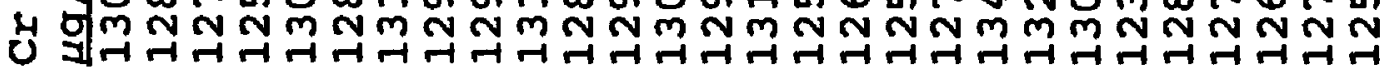

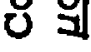




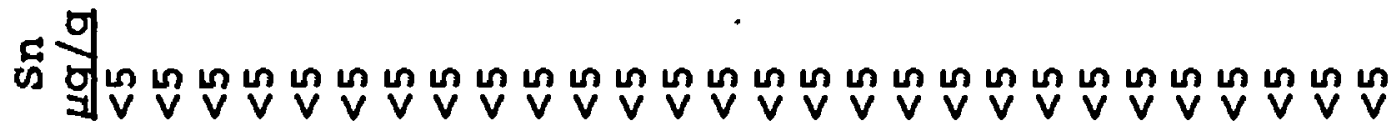

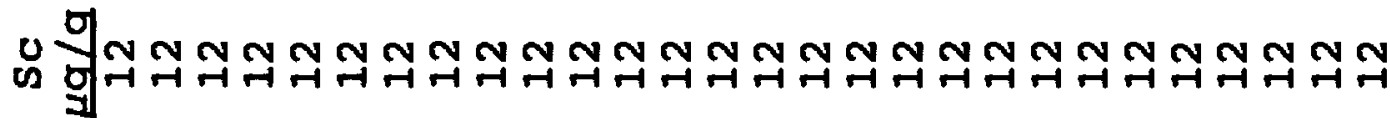

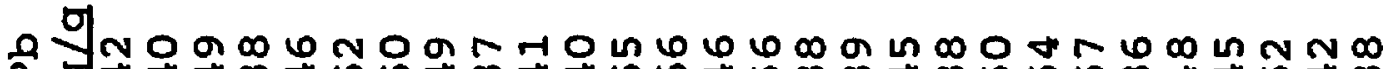

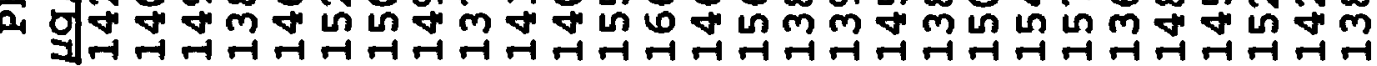

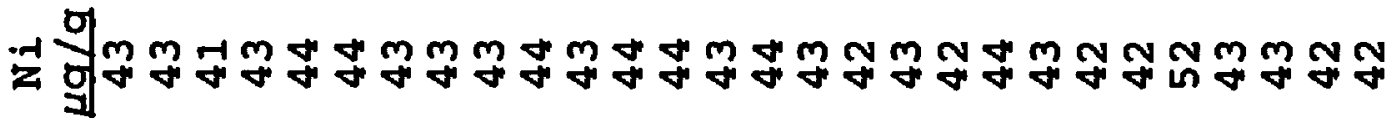

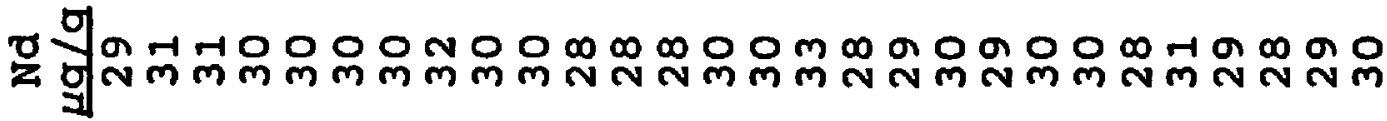

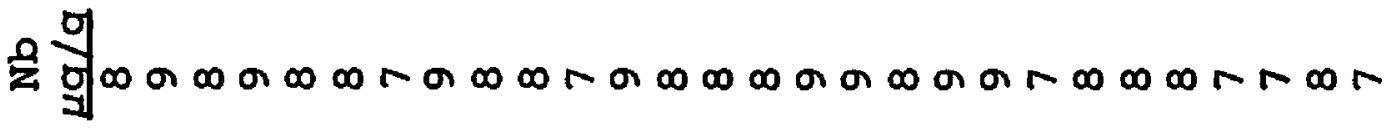

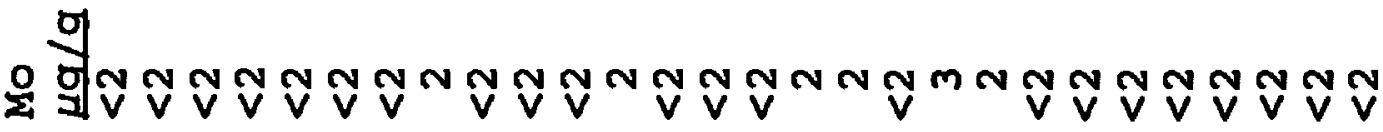

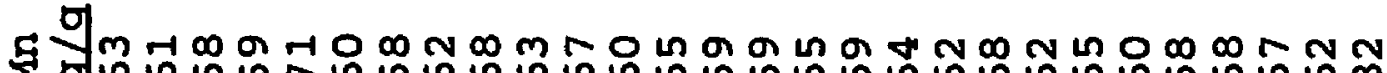

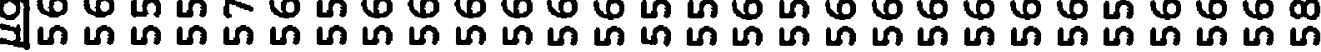

$m$

苟 ब م

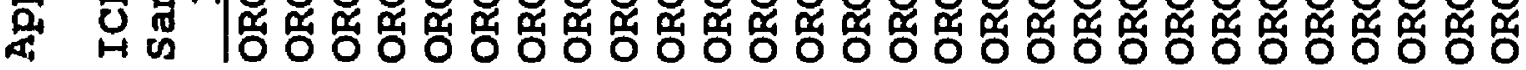




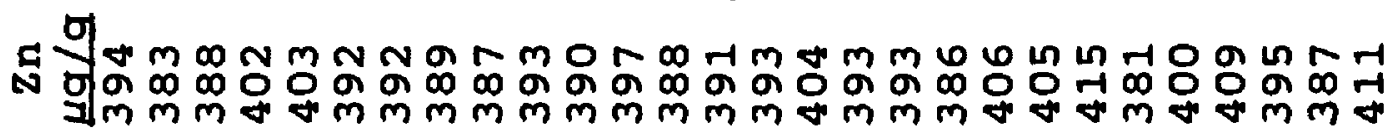

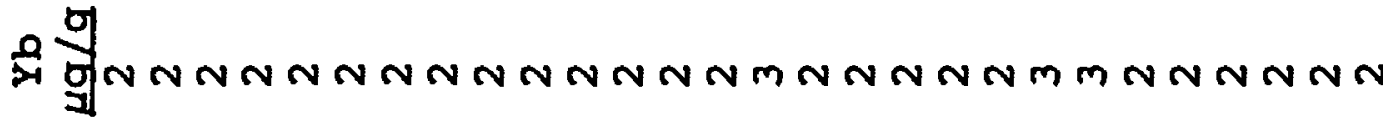

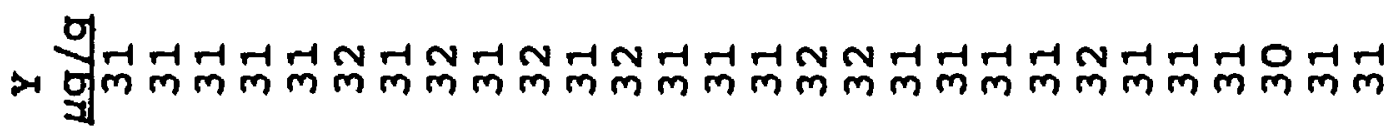

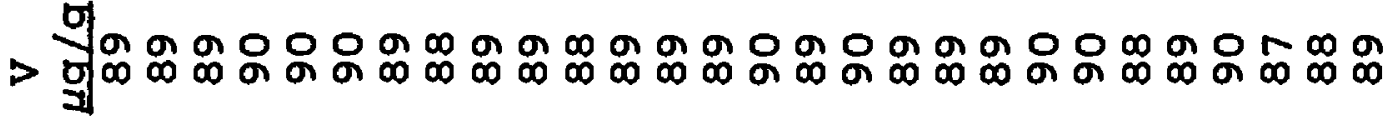

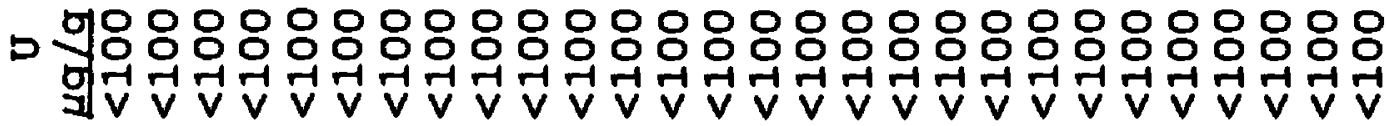

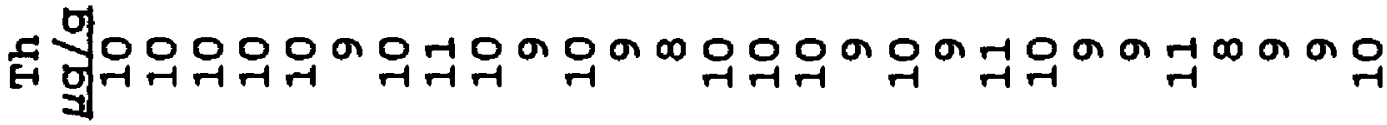

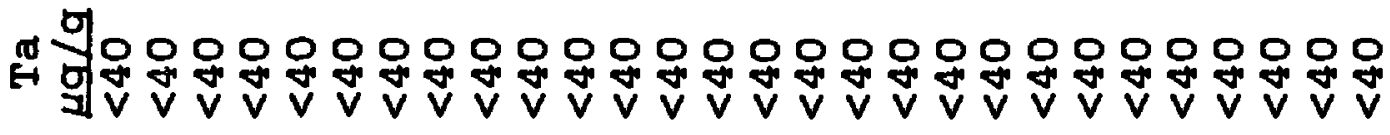

4 아 0 wo

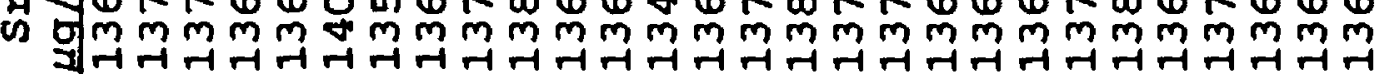

ต 西0 1 م.

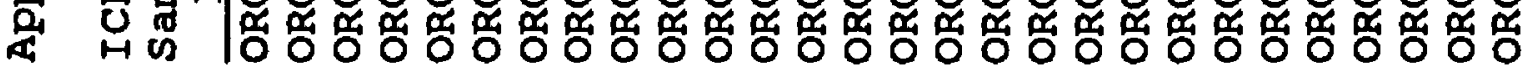




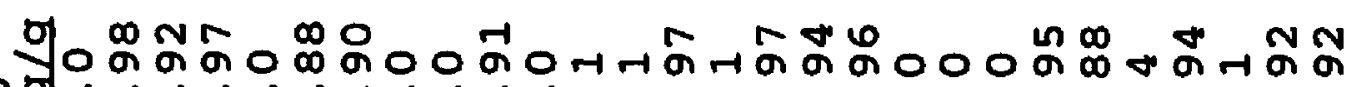

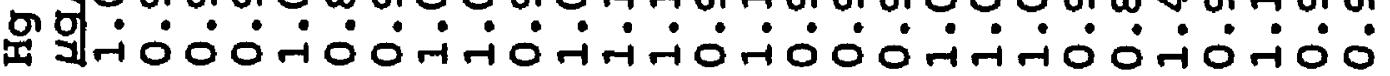

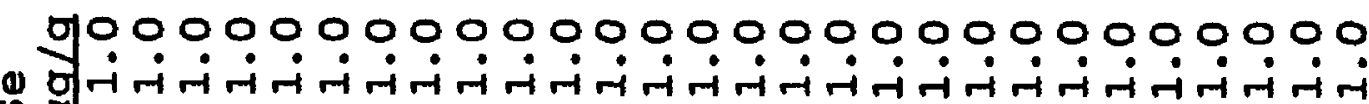
\&

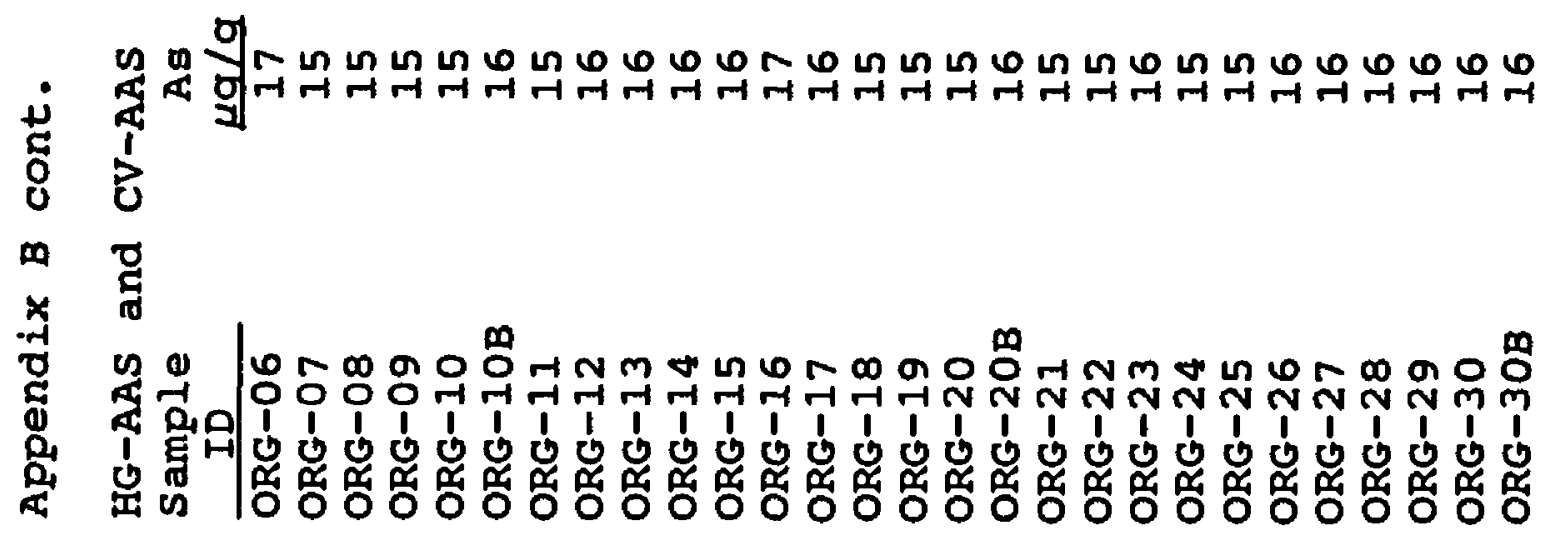




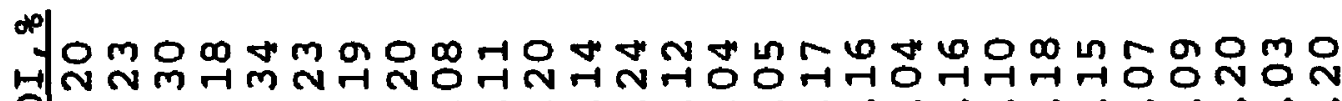

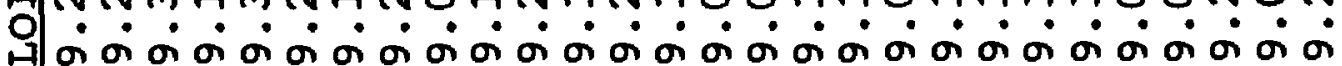

$+\infty$

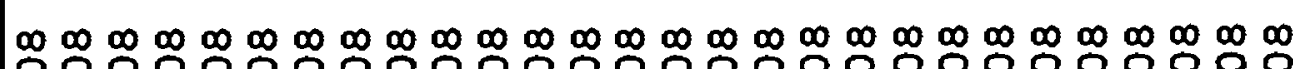

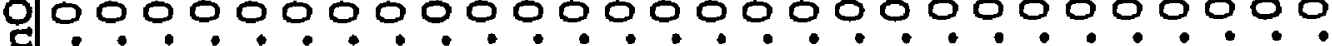

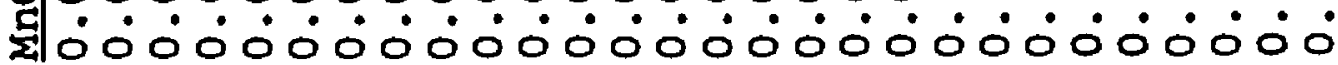

कูกุด

$m m \forall m \forall \forall \forall \forall m m \forall m m m m m m m m m \forall m \forall m \forall m m m$

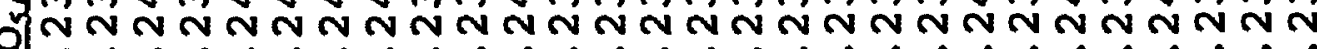

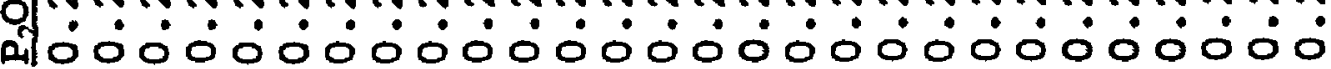

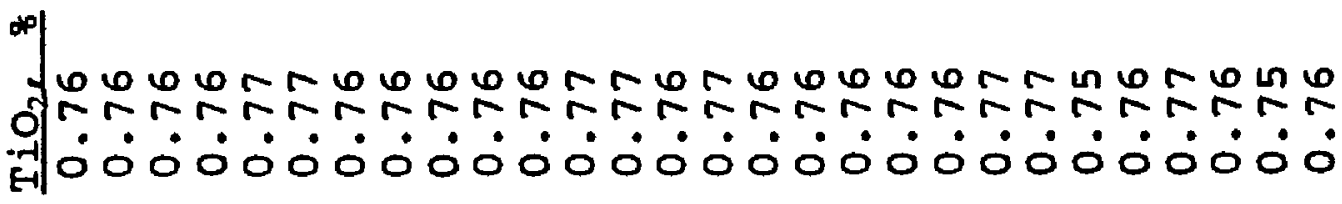

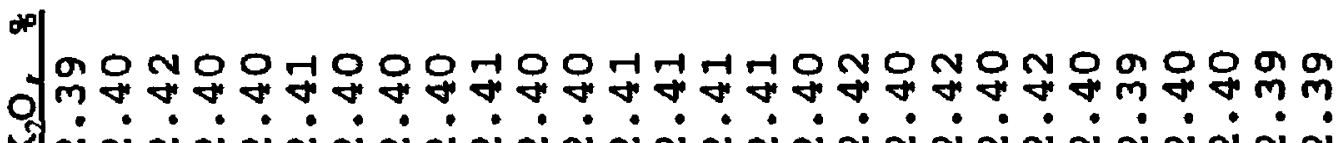

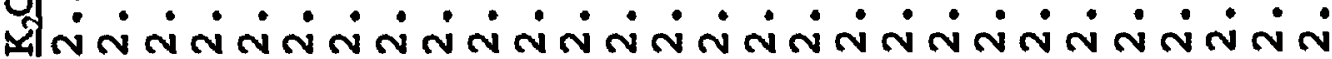

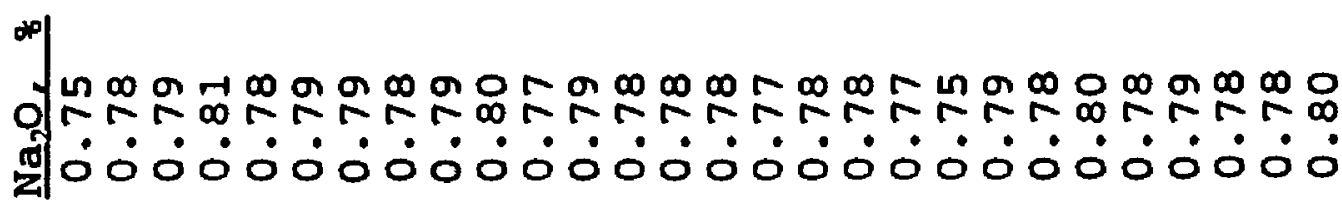

ชึ:

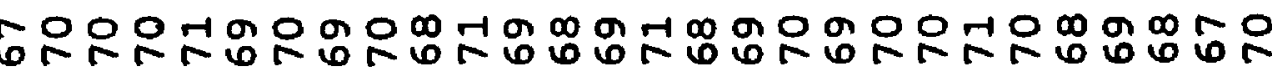

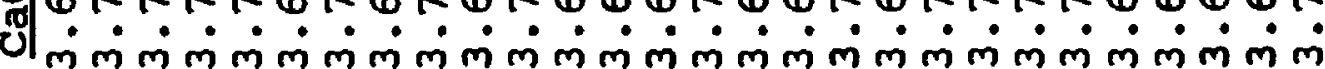

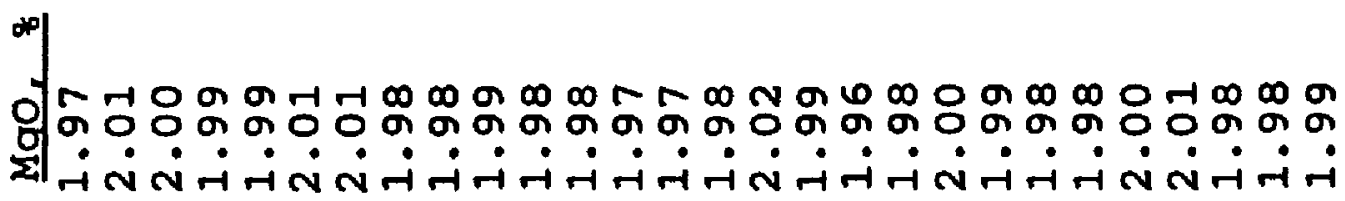

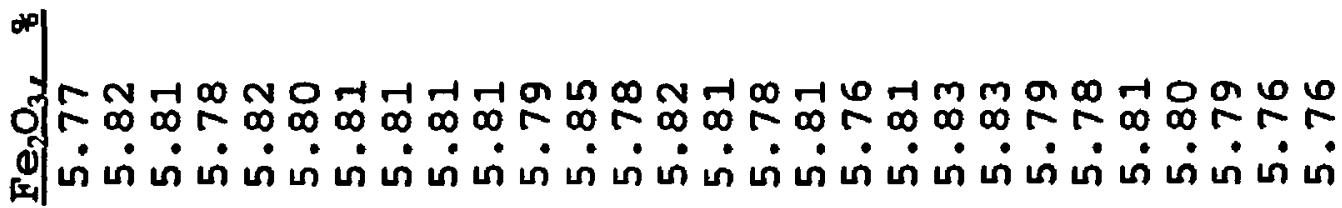

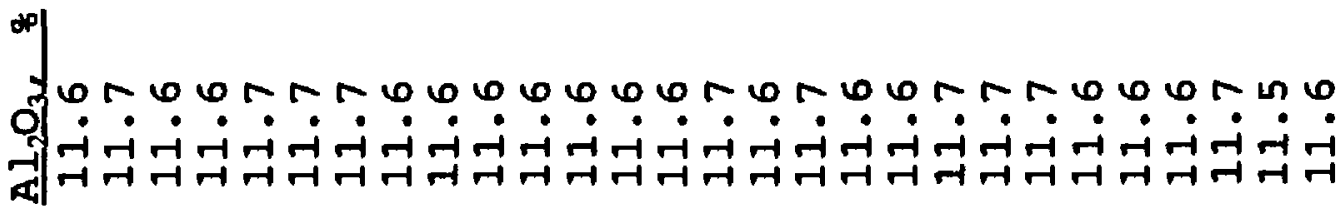

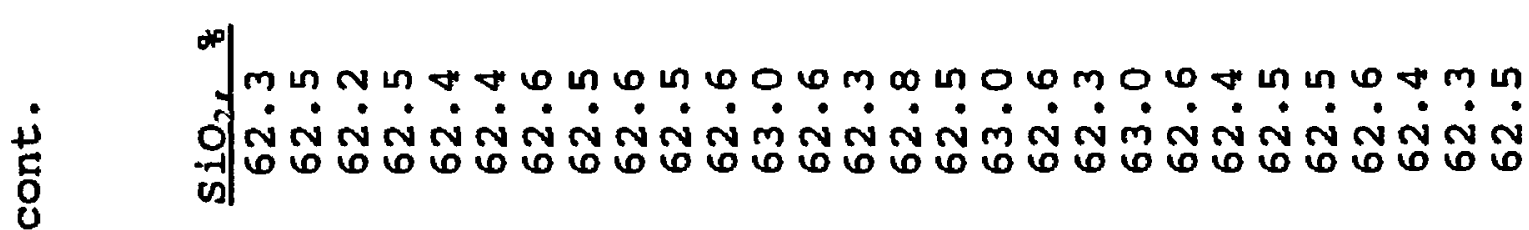

m

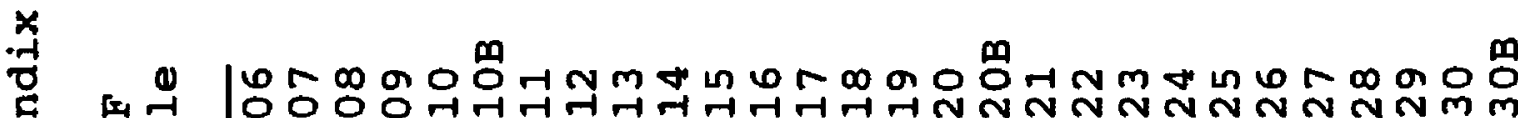
Q 


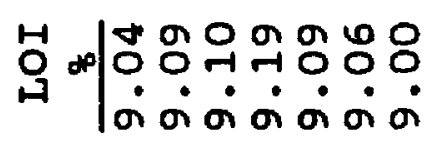

올ำ

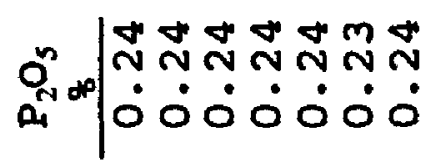

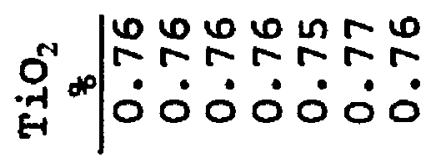

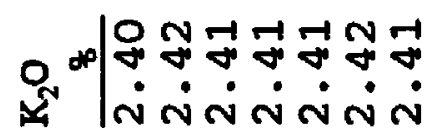

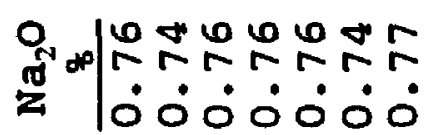

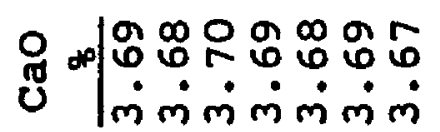

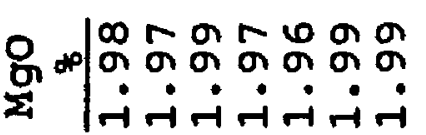

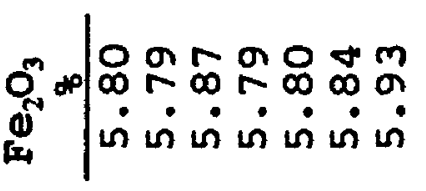

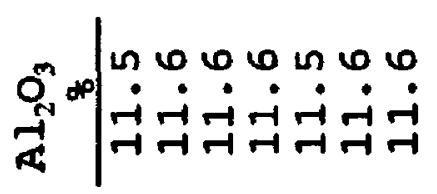

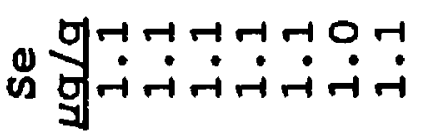

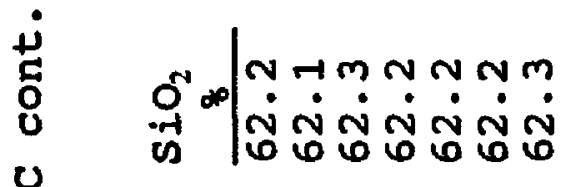

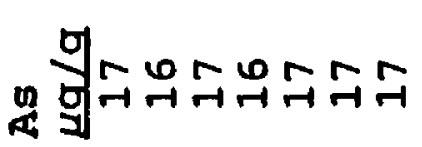

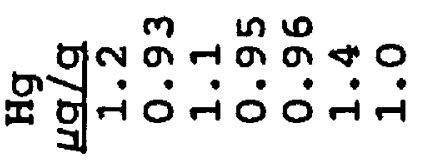


$\stackrel{-H}{E} \mid \begin{array}{lll}0 & m & m \\ m & m & m \\ \dot{0} & 0 & 0\end{array}$

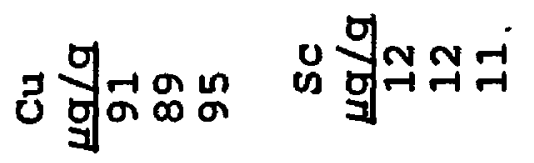

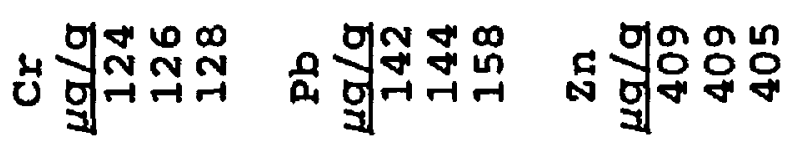

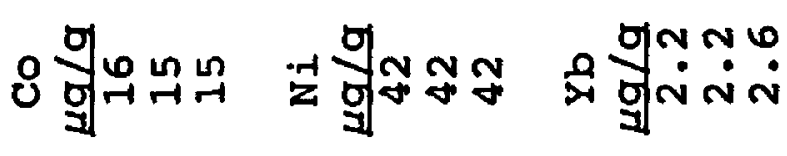

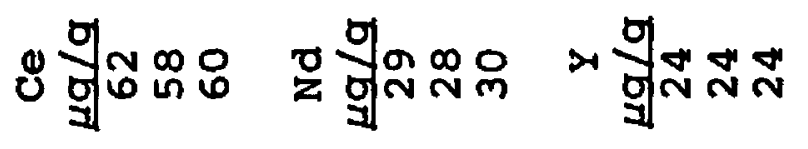

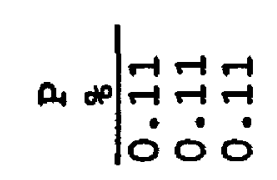

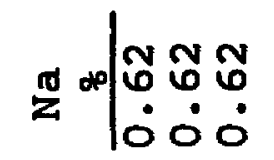

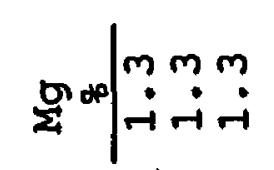

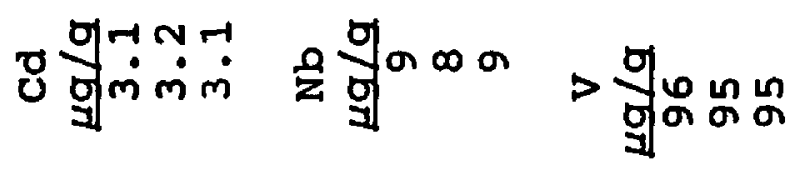

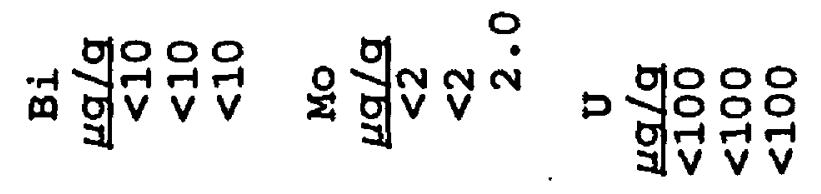

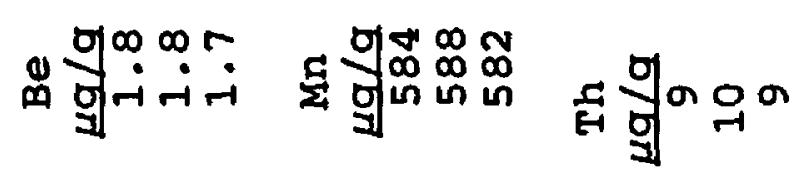

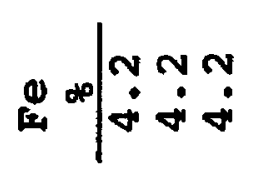

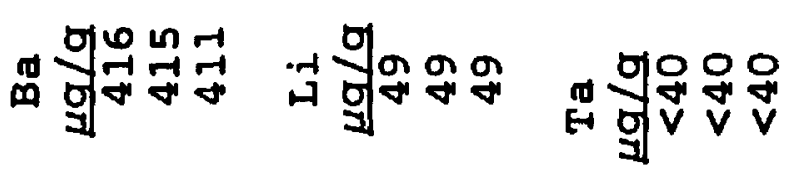

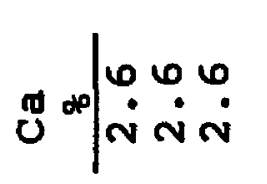

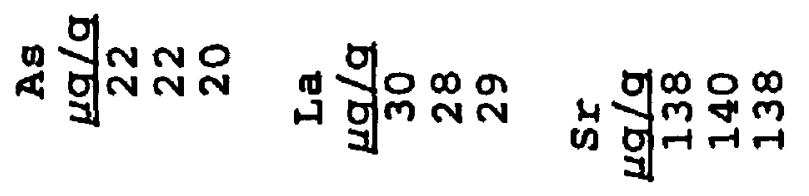

$\pi \mid \begin{array}{llll}0 & 0 & 0\end{array}$

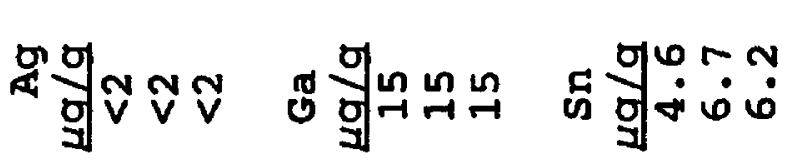

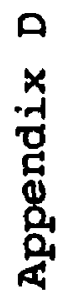

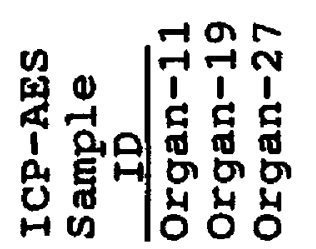

告|

年

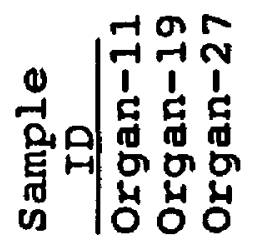

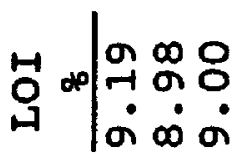

蛋

$\overbrace{n}^{n} \mid \begin{array}{lll}\pi & \infty & m \\ N & N & n \\ 0 & 0 & 0\end{array}$

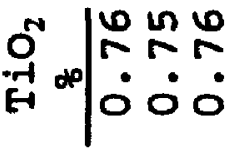

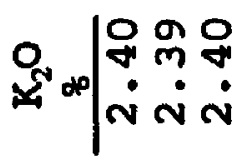

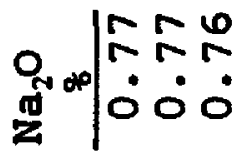

$N$

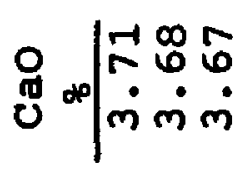

是 $\mid \begin{array}{lll}0 & m & n \\ 0 & \alpha & \alpha \\ \dot{v} & -1 & -1\end{array}$

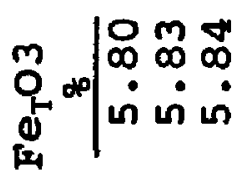

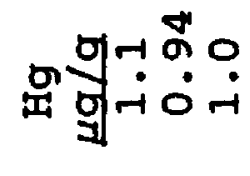

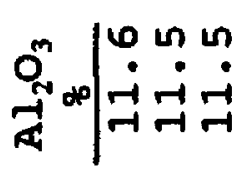

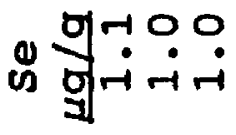

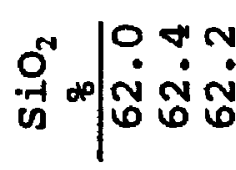

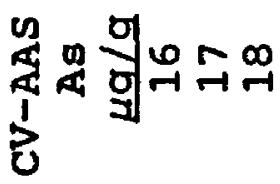

守 


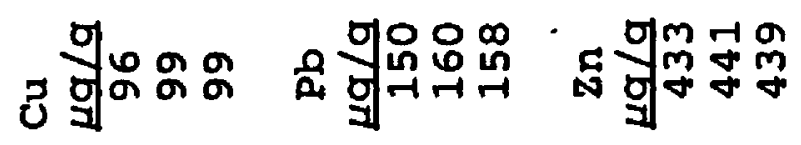

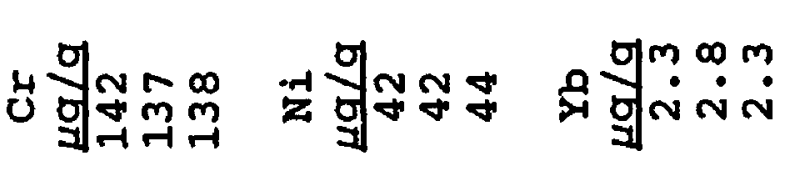

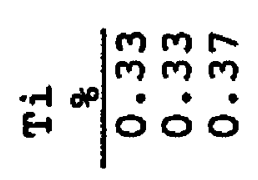

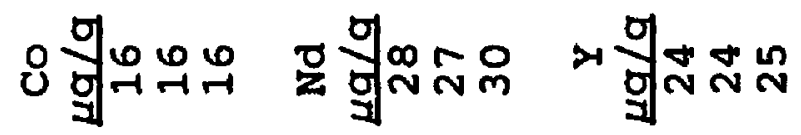

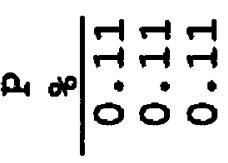

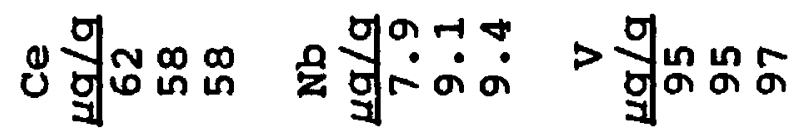
$\pi \mid \begin{array}{lll}\pi & 0 & 0 \\ 0 & 0 & 0 \\ 0 & 0 & 0\end{array}$

ชुํำं Ren

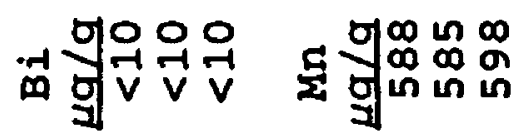

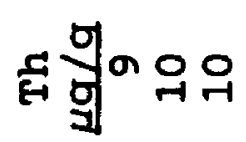

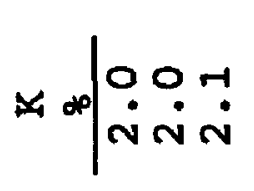

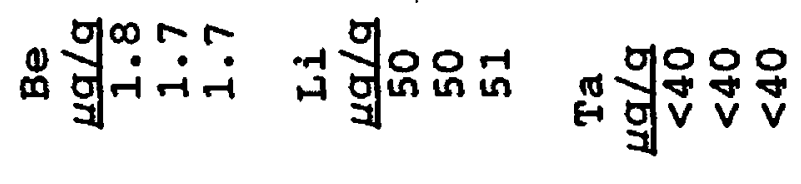
ঋ

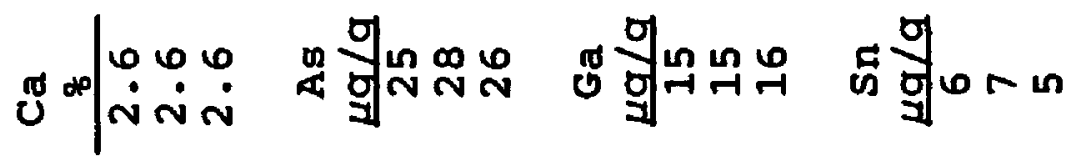

葋

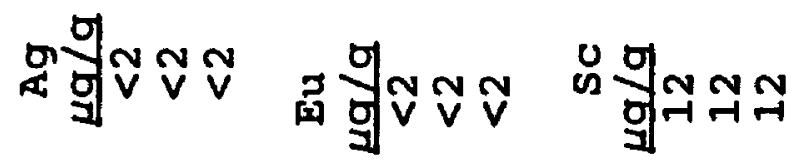

茯

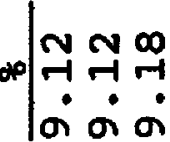
| 1000

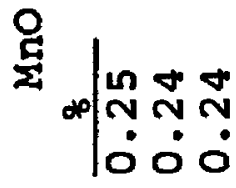

Oñ

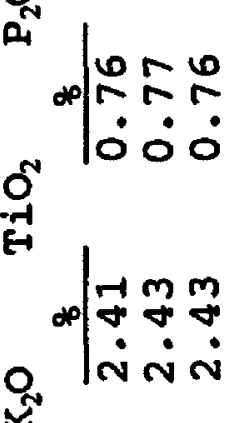

요

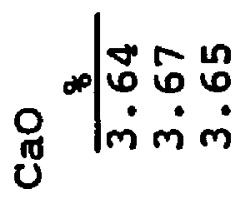

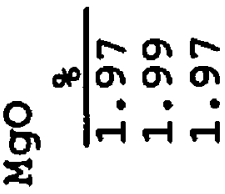

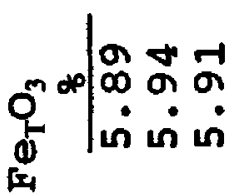

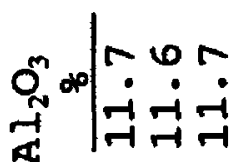

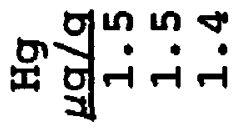

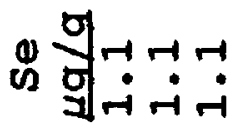

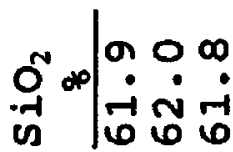

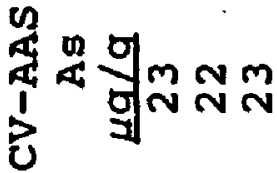

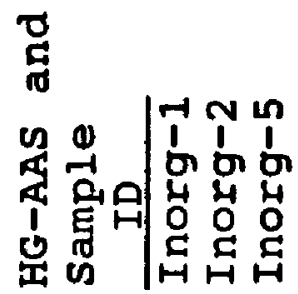

